\title{
SOME APPLICATIONS OF NON CONVEX SUBDIFFERENTIAL CALCULUS IN BINORMED SPACES
}

\author{
Jamal Hlal \\ ANO Laboratory, Faculty of Sciences \\ Mohammed First University \\ Oujda, 60000, MOROCCO
}

\begin{abstract}
In this article we are interested to give an analogue result of the subdifferentiation of the marginal functions in Banach spaces established by Mordukhovich and Shao in [6] using the so-called the srtong generalized limiting subdifferential defined in binormed space introduced by Hlal in [3].
\end{abstract}

AMS Subject Classification: 49J52, 49J50

Key Words: nonconvex calculus, strong limiting subdifferential, generalized normal cones, coderivy

\section{Introduction}

The study of subdifferentiability of marginal functions is of great interest not only because it is related to the Lagrange multipliers but also because it is connected to the study in sensitivity of some problems in optimization and optimal control. This class of functions are usually nonsmooth; therefore, to compute the generalized (in some sense) derivative for the marginal functions is a challenging issue which has many significations to various applications. We can see $[1,2,6]$.

We recall that the marginal function is defined by:

$$
m(x)=\inf \{\varphi(x, y) / y \in \Phi(x)\},
$$

where $\varphi: \mathrm{X} \times \mathrm{Y} \rightarrow \bar{R}$ an extended real-valued function and $\Phi$ a multifonction 
acting from $X$ into $Y$.

Mordukhovich and Shao [6] used the limiting subdifferential in order to prove formulas of subdifferentiation of marginal functions in general Banach spaces, however this result must involve the strict differentiability notion. The present paper studies, with the help of Mordukhovich's results is to generalize this interesting result for non necessarily Fréchet strictly differentiable mappings using the notion of limiting subdifferential in binormed spaces (generalized limiting subdifferential). Thus, our results are closely related to (extending in some sense) those of [6]. After recalling some definitions and notions in the first section, the second one is devoted to establish the main result of this paper related to subdifferential of marginal functions in binormed spaces. Finally, we give an example of a non Fréchet strictly differentiable mapping for which we can apply our theorem, while the theorem of Mordukhovich and Shao [6] cannot be used.

\section{Basic definitions and properties}

In this paper, $\left(\mathrm{X},\|\cdot\|_{1},\|\cdot\|_{2}\right)$ and $\left(Y,\|\cdot\|_{3},\|\cdot\|_{4}\right)$ denote two binormed spaces such that $\left(X,\|\cdot\|_{2}\right)$ and $\left(Y,\|\cdot\|_{4}\right)$ are two separable Banach spaces, and for some $c>0, c^{\prime}>0\|\cdot\|_{1} \leq c\|\cdot\|_{2},\|\cdot\|_{3} \leq c^{\prime}\|\cdot\|_{4}$. For $(x, y) \in X \times Y$ we set $\|(x, y)\|_{5}=\|x\|_{1}+\|y\|_{3}$ and $\|(x, y)\|_{6}=\|x\|_{2}+\|y\|_{4}$. Hence, $X \times Y$ becomes a binormed space under the pair of norms $\left(\|\cdot\|_{5},\|\cdot\|_{6}\right)$. Moreover, $\|\cdot\|_{5} \leq \max \left(c, c^{\prime}\right)\|\cdot\|_{6}$.

By $C l^{1} \Omega$ we denote the closure of $\Omega$ in $\left(X, \|\right.$. $\left.\|_{1}\right)$, while $x \stackrel{\varphi,\|\cdot\|_{1}}{\rightarrow} \bar{x}$ (respectively $x \stackrel{\Omega,\|\cdot\|_{1}}{\longrightarrow} \bar{x}$ ) means that $x \stackrel{\|\cdot\|_{1}}{\rightarrow} \bar{x}$ with $\varphi(x) \rightarrow \varphi(\bar{x})$ (respectively, $x \rightarrow \bar{x}$ with respect to the norm $\|\cdot\|_{1}$ and $x, \bar{x} \in \Omega$ ). Let $B_{\delta}^{1}(\bar{x})$ denotes the open ball centered at $\bar{x}$ with radius $\delta$ in $\left(X,\|\cdot\|_{1}\right)$, where $\bar{x} \in X$ and $\delta>0$. For any multifunction $\Phi$ acting from $X$ into its topological dual $\left(X,\|\cdot\|_{2}\right)^{*}$, we define the $(1,2)$ $\left(\|\cdot\|_{1},\|\cdot\|_{2}\right)$-sequential Kuratowski-Painlevé upper limit by: $\lim _{x \rightarrow \bar{x}} \sup \Phi(x) \equiv$ $\left(\|\cdot\|_{1},\|\cdot\|_{2}\right)-\lim \sup _{x \rightarrow \bar{x}} \Phi(x)=\left\{x^{*} \in\left(X,\|\cdot\|_{2}\right)^{*} / \exists x_{k}^{*} \stackrel{\omega^{*}}{\rightarrow} x^{*}, \exists x_{k} \stackrel{\|\cdot\|_{1}}{\rightarrow} \bar{x}\right.$ with $\left.\quad x_{k}^{*} \in \Phi\left(x_{k}\right) \quad \forall k=1,2, \ldots\right\}$, where $\stackrel{\omega^{*}}{\rightarrow}$ denotes the convergence for the weak-star topology $* \sigma\left(\left(X,\|\cdot\|_{2}\right)^{*}, X\right)$ of $\left(X,\|\cdot\|_{2}\right)^{*}$.

Definition 1. ([4]) Let $U$ be an open subset of $\left(X,\|\cdot\|_{1}\right)$ and let $\varphi$ : $U \longrightarrow R$ be a real-valued function. Assume that $\bar{x} \in U$.

We say that $\varphi$ is $\left(\|\cdot\|_{1},\|\cdot\|_{2}\right)$-strictly Fréchet differentiable at $\bar{x}$ if there 
exists a continuous linear operator $\mathrm{L}$ from $\left(X,\|.\|_{2}\right)$ into $R$ such that

$$
\lim _{u, x \rightarrow \|_{1} \bar{x}} \frac{\varphi(u)-\varphi(x)-L(u-x)}{\|u-x\|_{2}}=0 .
$$

In this case, we set $\nabla^{1,2} \varphi(x) \equiv\left(\|\cdot\|_{1},\|\cdot\|_{2}\right)-\nabla \varphi(x)=L$.

Let us remark that if $\varphi$ is $\left(\|\|_{1},\|\cdot\|_{2}\right)$-strictly Fréchet differentiable at $\bar{x}$, then it is necessarily $\|\cdot\|_{2}$-strictly Fréchet differentiable at $\bar{x}$ and therefore, continuous with respect to the norm $\|\cdot\|_{2}$. But in general, even if $\varphi$ is $\left(\|\cdot\|_{1}\right.$ , \|\|$_{2}$ )-strictly Fréchet differentiable at $\bar{x}$, it is not necessarily $\|.\|_{1}$-strictly Fréchet differentiable at $\bar{x}$. But the converse is true, that is, if $\varphi$ is \|\|$_{1}$-strictly Fréchet differentiable at $\bar{x}$, then it is $\left(\|\|_{1},\|\cdot\|_{2}\right)$-strictly Fréchet differentiable at $\bar{x}$.

Definition 2. ([4]) Let $\varepsilon \geq 0$. We define the generalized Fréchet $\varepsilon$ subdifferential of $\varphi: X \longrightarrow R$ at $x \in \operatorname{dom} \varphi$ with respect to the pair of norms (\| . $\left.\left\|_{1},\right\| \cdot \|_{2}\right)$ by: $\hat{\partial}_{\varepsilon}^{1,2} \varphi(x)=\left\{x^{*} \in\left(X,\|\cdot\|_{2}\right)^{*} \liminf _{u \stackrel{\|\cdot\|_{1}}{\longrightarrow} x} \frac{\varphi(u)-\varphi(x)-\left\langle x^{*}, u-x\right\rangle}{\|u-x\|_{2}} \geq\right.$ $-\varepsilon\}$. If $\varepsilon=0$ this construction is called the generalized presubdifferential or generalized Fréchet subdifferential of $\varphi$ at $x$ with respect to the pair of norms $\left(\|\cdot\|_{1},\|\cdot\|_{2}\right)$ and we denoted it by $\hat{\partial}^{1,2} \varphi(x)$.

Definition 3. ([3]) Let $\varphi: X \longrightarrow \bar{R}$ be an extended-real-valued function and $\bar{x} \in \operatorname{dom} \varphi$. Assume that $\varphi$ is l.s.c. around $\bar{x}$ with respect to the norm $\|\cdot\|_{1}$.

We define the strong generalized limiting subdifferential $\breve{\partial}^{1,2} \varphi(\bar{x})$ with respect to the pair of norms $\left(\|\cdot\|_{1},\|\cdot\|_{2}\right)$ by:

$$
\breve{\partial}^{1,2} \varphi(\bar{x})=\limsup _{\rho \searrow 0, \varepsilon \searrow 0, x,\|l\| \|_{11}, \rho} \hat{\partial}_{\bar{x}}^{1,2} \varphi(x),
$$

where, $\quad \limsup \hat{\partial}_{\varepsilon}^{1,2} \varphi(x)$ denotes the strong sequential $\rho \searrow 0, \varepsilon \backslash 0, x \cdot \stackrel{\|\cdot\|_{1} f, \rho}{\rightarrow} \bar{x}$

Kuratowski-Painlevé upper limit with respect to the pair of norms $\left(\|\|_{1}\right.$, $\left.\|\cdot\|_{2}\right)$, i.e.:

$\limsup \quad \hat{\partial}_{\varepsilon}^{1,2} \varphi(x)=\left\{x^{*} \in\left(X,\|\cdot\|_{2}\right)^{*} / \exists \varepsilon_{k} \downarrow 0, \exists \rho_{k} \downarrow 0, \exists x_{k} \stackrel{\|\cdot\|_{1}, \varphi}{\rightarrow}\right.$ $\rho \searrow 0, \varepsilon \searrow 0, x^{\|\cdot\|_{1}, \varphi, \rho} \overline{\bar{x}}$

$\bar{x}, \exists x_{k}^{*} \stackrel{\omega^{*}}{\rightarrow} x^{*}$ such that $\left(\rho_{k}\right)_{k}$ is a nonincreasing sequence satisfying: $\rho_{k}>$ 
0 and $\forall\left(k_{1}, k_{2}\right)$ there is a positive integer $k_{3} \geq k_{2}$ such that

$$
\begin{gathered}
\forall x^{\prime} \in B_{\rho_{k_{1}}}^{1}\left(x_{k_{3}}\right) \quad \varphi\left(x^{\prime}\right)-\varphi\left(x_{k_{3}}\right)-\left\langle x_{k_{3}}^{*}, x^{\prime}-x_{k_{3}}\right\rangle \geq-2 \varepsilon_{k_{3}}\left\|x^{\prime}-x_{k_{3}}\right\|_{2} \\
\text { and } \left.\left\|\bar{x}-x_{k_{3}}\right\|_{1}<\frac{\rho_{k_{3}}}{2}\right\} .
\end{gathered}
$$

Let us remark that in general, $\breve{\partial}^{1,2} \varphi(\bar{x}) \not \subset \breve{\partial}^{2} \varphi(\bar{x})$ and $\breve{\partial}^{2} \varphi(\bar{x}) \not \subset \breve{\partial}^{1,2} \varphi(\bar{x})$. But if $\|.\|_{1}$ is equivalent to $\|$. $\|_{2}$, then, $\breve{\partial}^{1} \varphi(\bar{x})=\breve{\partial}^{2} \varphi(\bar{x})=\breve{\partial}^{1,2} \varphi(\bar{x})$. So, the generalized strong limiting subdifferential is a generalized version of the strong limiting subdifferential in normed space defined by Lahrech et al. in [5].

Definition 4. Let $\bar{x} \in C l^{1} \Omega$, the set : $\breve{N}^{1,2}(\bar{x}, \Omega)=\breve{\partial}^{1,2} \delta(\bar{x}, \Omega)$ is called the strong generalized normal cone to $\Omega$ at $\bar{x}$ with respect to the pair of norms $\left(\|\cdot\|_{1},\|\cdot\|_{2}\right)$. We set $\breve{N}^{1,2}(\bar{x}, \Omega)=\emptyset$ for $\bar{x} \notin C l^{1} \Omega$. Where $\delta(., \Omega)$ the indicator function defined by: $\delta(x, \Omega)=0$ if $x \in \Omega$ and $\delta(x, \Omega)=\infty$ if $x \notin \Omega$.

Denote by $\breve{N}^{2}(\bar{x}, \Omega)$ the strong normal cone to $\Omega$ at $\bar{x}$ with respect to the norm $\|$. $\|_{2}$. In general, if $x \in C l^{2} \Omega$, then $\breve{N}^{1,2}(\bar{x}, \Omega) \not \subset \breve{N}^{2}(\bar{x}, \Omega)$ and $N^{2}(\bar{x}, \Omega) \not \subset \breve{N}^{1,2}(\bar{x}, \Omega)$. But, if $\|$. $\|_{1}$ is equivalent to $\|$. $\|_{2}$, then $\breve{N}^{1,2}(\bar{x}, \Omega)=\breve{N}^{1}(\bar{x}, \Omega)=N^{2}(\bar{x}, \Omega)$. So, our strong generalized normal cone $\breve{N}^{1,2}(\bar{x}, \Omega)$ defined in binormed space $\left(X,\|\cdot\|_{1},\|\cdot\|_{2}\right)$ generalizes in some sens the notion of classical normal cone defined in normed space.

Definition 5. ([2]) Let $\Phi$ a multifonction acting from $\left(X,\|\cdot\|_{1},\|\cdot\|_{2}\right)$ into $\left(Y,\|.\|_{3},\|.\|_{4}\right)$ and let $(\bar{x}, \bar{y}) \in \operatorname{cl} g p h \Phi$. The multifonction $\mathrm{D}^{* 1,2} \Phi(\bar{x}, \bar{y})$ acting from $\left(Y, \| \text {. } \|_{4}\right)^{*}$ into $\left(X\|.\|_{2}\right)^{*}$ defined by: $D^{* 1,2} \Phi(\bar{x}, \bar{y})\left(y^{*}\right)=\left\{x^{*} \in\right.$ $\left.\left(X,\|\cdot\|_{2}\right)^{*} /\left(x^{*},-y^{*}\right) \in \breve{N}^{1,2}((\bar{x}, \bar{y}) ; g p h \Phi)\right\}$ is called the generalized coderivy of $\Phi$ at $(\bar{x}, \bar{y})$ with respect to the pair of norms $\left(\|.\|_{1},\|.\|_{2}\right)$.

We set $D^{* 1,2} \Phi(\bar{x}, \bar{y})\left(y^{*}\right)=\emptyset$ if $(\bar{x}, \bar{y}) \notin \operatorname{cl} \operatorname{gph} \Phi$.

Let us remark that in general, $D^{* 1,2} \Phi(\bar{x}, \bar{y})\left(y^{*}\right) \not \subset D^{* 2} \Phi(\bar{x}, \bar{y})\left(y^{*}\right)$ and $D^{* 2} \Phi(\bar{x}, \bar{y})\left(y^{*}\right) \not \subset D^{* 1,2} \Phi(\bar{x}, \bar{y})\left(y^{*}\right)$. But if $\|$. $\|_{1}$ is equivalent to $\|$. $\|_{2}$, then $D^{* 1} \Phi(\bar{x}, \bar{y})\left(y^{*}\right)=D^{* 2} \Phi(\bar{x}, \bar{y})\left(y^{*}\right)=D^{* 1,2} \Phi(\bar{x}, \bar{y})\left(y^{*}\right)$. So, the generalized coderivy is a generalized version of the coderivy in [6]

Definition 6. ([6]) We say that the multifunction $M$ acting from $\mathrm{X}$ into $\mathrm{Y}$ have the lower semicompactness property with respect to the pair of norms $\left(\|\cdot\|_{1},\|\cdot\|_{3}\right)$ around the point $\bar{x}$ if there exists un \|\|$_{1}$-neighborhood $U$ of $\bar{x}$ 
such that for any $x \in \mathrm{U}$ and any sequence $x_{k} \stackrel{\|\cdot\|_{1}}{\rightarrow} x$ as $k \rightarrow \infty$ there is a sequence $y_{k} \in M\left(x_{k}\right) \mathrm{k}=1.2 \ldots$ which contains a subsequence converging in $\left(Y,\|\cdot\|_{3}\right)$.

\section{Subdifferentiation of marginal functions in terms of the strong generalized limiting subdifferential}

The following theorem is the main result of this paper. Before that, we need the set

$$
M(x)=\{y \in \Phi(x) / \varphi(x, y)=m(x)\}
$$

associated with (1).

Theorem 7. Let $\Phi: X \Rightarrow Y$ have closed graph, let $M$ in (4) be lower semicompact around $\bar{x} \in$ dom $m$ with respect to the pair of norms $\left(\|\|_{1}, \|\right.$ . $\left.\|_{3}\right)$, and let $\varphi$ be $\|$. $\|_{5}$-l.s.c. on gph $\Phi$ and $\left(\|\right.$. $\left\|_{5},\right\|$. $\left.\|_{6}\right)$-Fréchet strictly differentiable at $(\bar{x}, \bar{y})$ for any $\bar{y} \in M(\bar{x})$. Then:

$$
\breve{\partial}^{1,2} m(\bar{x}) \subset \bigcup_{\bar{y} \in M(\bar{x})}\left[\nabla_{x}^{1,2} \varphi(\bar{x}, \bar{y})+D^{* 5,6} \Phi(\bar{x}, \bar{y})\left(\nabla_{y}^{3,4} \varphi(\bar{x}, \bar{y})\right)\right] .
$$

Proof. First we check that the marginal function is l.s.c. around $\bar{x}$ under the assumptions made with respect to the norm $\|.\|_{1}$ indeed, let $U$ be a neighborhood of $\bar{x}$ with respect to the norm $\|\cdot\|_{1}$ from the local semicompactness for $M$. Taking any $x \in U$ and sequence $x_{k} \stackrel{\|\cdot\|_{1}}{\rightarrow} x$ as $k \rightarrow \infty$, we find a sequence $y_{k} \in M\left(x_{k}\right)$ that contains a subsequence convergent to some point $y \in Y$ with respect to the norm $\|\cdot\|_{3}$ and $(x, y) \in g p h \Phi$. Since $\varphi$ is $\|\cdot\|_{5}$ l.s.c.on gph $\Phi$, then: $m(x) \leq \varphi(x, y) \leq \liminf _{k \rightarrow \infty} \Phi\left(x_{k}, y_{k}\right)=\liminf _{k \rightarrow \infty} m\left(x_{k}\right)$ that proves the l.s.c. of $m$ around $x$. Now let us consider a function $f: X \times Y \rightarrow R$ defined by:

$$
f(x, y)=\varphi(x, y)+\delta((x, y), g p h \Phi)
$$

and let us prove that

$$
\breve{\partial}^{1,2} m(\bar{x}) \subset\left\{x^{*} \in\left(X,\|\cdot\|_{2}\right)^{*} /\left(x^{*}, 0\right) \in \breve{\partial}^{5,6} f(\bar{x}, \bar{y}), \bar{y} \in M(\bar{x})\right\} .
$$

Let $x^{*} \in \breve{\partial}^{1,2} m(\bar{x})$, then there exists sequences $x_{k} \stackrel{\|\cdot\|_{1}}{\rightarrow} \bar{x}, x_{k}^{*} \stackrel{\omega^{*}}{\rightarrow} x^{*}, \epsilon_{k} \searrow 0$ such that $m\left(x_{k}\right) \rightarrow m(\bar{x})$ as, $k \rightarrow \infty$ and $x_{k}^{*} \in \breve{\partial}_{\epsilon_{k}}^{1,2} m\left(x_{k}\right)$ for all $k=1,2, \ldots$ Therefore, there exists a sequence $\rho_{k} \searrow 0$ as $k \rightarrow \infty$ with $\left\langle x_{k}^{*}, x-x_{k}\right\rangle \leq$ 
$m(x)-m\left(x_{k}\right)+2 \epsilon_{k}\left\|x-x_{k}\right\|_{2} \forall x \in B_{\rho_{k}}^{1}\left(x_{k}\right), k=1,2, \ldots$. By definitions of $m$ and $M$, for any $y_{k} \in M\left(x_{k}\right)$ we have: $\left\langle\left(x_{k}^{*}, 0\right),(x, y)-\left(x_{k}, y_{k}\right)\right\rangle \leq f(x, y)-$ $f\left(x_{k}, y_{k}\right)+2 \rho_{k}\left(\left\|x-x_{k}\right\|_{2}+\left\|y-y_{k}\right\|_{4}\right)$ for all $(x, y) \in B_{\rho_{k}}^{5}\left(x_{k}, y_{k}\right), k=1,2, \ldots$ Due to (3) and (5) we deduce that:

$$
\left(x_{k}^{*}, 0\right) \in \breve{\partial}_{2 \epsilon_{k}}^{5,6} f\left(x_{k}, y_{k}\right) \forall y_{k} \in M\left(x_{k}\right) k=1,2, \ldots
$$

Using the lower semicompactness of $M$ around $\bar{x}$, we can select sequence $y_{k} \in$ $M\left(x_{k}\right)$ which contains a subsequence convergent to some point $\bar{y} \in \Phi(\bar{x})$ with respect to the norm $\|\cdot\|_{3}$, then $\varphi\left(x_{k}, y_{k}\right)=m\left(x_{k}\right)$. On the other hand,

implies that

$$
m(\bar{x}) \leq \varphi(\bar{x}, \bar{y}) \leq \liminf _{\left(x_{k}, y_{k}\right) \stackrel{\|\cdot\|_{5}}{\rightarrow}(\bar{x}, \bar{y})} \varphi\left(x_{k}, y_{k}\right)=\liminf _{x_{k} \stackrel{\|_{1}}{\rightarrow} \bar{x}} m\left(x_{k}\right)=m(\bar{x}) \text {, and this }
$$

$\varphi(\bar{x}, \bar{y})=m(\bar{x})$, i.e. $\bar{y} \in M(\bar{x})$ and $f\left(x_{k}, y_{k}\right) \rightarrow \varphi(\bar{x}, \bar{y})$ as $k \rightarrow \infty$ and by applying (3) and (7) we conclude that $\left(x^{*}, 0\right) \in \breve{\partial}^{5,6} f(\bar{x}, \bar{y})$ which proves $(6)$. Applying Theorem 4 from [4] for $f$, we have:

$$
\breve{\partial}^{5,6} f(\bar{x}, \bar{y})=\left(\nabla_{x}^{1,2} \varphi(\bar{x}, \bar{y}), \nabla_{y}^{3,4} \varphi(\bar{x}, \bar{y})\right)+\breve{\partial}^{5,6} \delta((\bar{x}, \bar{y}), g p h \Phi) .
$$

Then,

$$
\breve{\partial}^{5,6} f(\bar{x}, \bar{y})=\left(\nabla_{x}^{1,2} \varphi(\bar{x}, \bar{y}), \nabla_{y}^{3,4} \varphi(\bar{x}, \bar{y},)\right)+\breve{N}^{5,6}((\bar{x}, \bar{y}), g p h \Phi)
$$

Since $\left(x^{*}, 0\right) \in \breve{\partial}^{5,6} f(\bar{x}, \bar{y}), \bar{y} \in M(\bar{x})$, then:

$$
\left(x^{*}, 0\right) \in\left(\nabla_{x}^{1,2} \varphi(\bar{x}, \bar{y}), \nabla_{y}^{3,4} \varphi(\bar{x}, \bar{y},)\right)+\breve{N}^{5,6}((\bar{x}, \bar{y}), g p h \Phi), \quad \bar{y} \in M(\bar{x})
$$

i.e: $\left(x^{*}-\nabla_{x}^{1,2} \varphi(\bar{x}, \bar{y}),-\nabla_{y}^{3,4} \varphi(\bar{x}, \bar{y})\right) \in \breve{N}^{5,6}((\bar{x}, \bar{y}), g p h \Phi)$. Applying the definition of the coderivy we deduct that:

$$
x^{*}-\nabla_{x}^{1,2} \varphi(\bar{x}, \bar{y}) \in \breve{D}^{* 5,6} \Phi(\bar{x}, \bar{y})\left(\nabla_{y}^{3,4} \varphi(\bar{x}, \bar{y})\right), \quad \bar{y} \in \varphi(\bar{x})
$$

this implies that:

$$
x^{*} \in \nabla_{x}^{1,2} \varphi(\bar{x}, \bar{y})+\breve{D}^{* 5,6} \Phi(\bar{x}, \bar{y})\left(\nabla_{y}^{3,4} \varphi(\bar{x}, \bar{y})\right), \quad \bar{y} \in \varphi(\bar{x})
$$

thus $\breve{\partial}^{1,2} m(\bar{x}) \subset \nabla_{x}^{1,2} \varphi(\bar{x}, \bar{y})+\breve{D}^{* 5,6} \Phi(\bar{x}, \bar{y})\left(\nabla_{y}^{3,4} \varphi(\bar{x}, \bar{y})\right)$ and we arrive at

$$
\breve{\partial}^{1,2} m(\bar{x}) \subset \bigcup_{\bar{y} \in M(\bar{x})}\left[\nabla_{x}^{1,2} \varphi(\bar{x}, \bar{y})+D^{* 5,6} \Phi(\bar{x}, \bar{y})\left(\nabla_{y}^{3,4} \varphi(\bar{x}, \bar{y})\right)\right] .
$$


Finally we give an example of a non Fréchet strictly differentiable mapping with respect to the norm $\|\cdot\|_{1}$ for which we can apply our theorem (Theorem 7 ), while the theorem from [6] given by Mordukhovich and Shao cannot be used. Let $\Omega$ be a bounded domain in $I R^{2}$ and let $X=W_{0}^{1,2}(\Omega)$ be the Sobolev space under its usual norm $\|\cdot\|_{2}=\|\cdot\|_{W_{0}^{1,2}(\Omega)}$. Let also $p$ and $\varepsilon$ be such that $0<\varepsilon<1, \varepsilon+2<p<\infty$. Set $\|\cdot\|_{1}=\|\cdot\|_{L^{p}(\Omega)}$. Remark that $\left(X,\|\cdot\|_{2}\right)$ is a Banach separable space. Since $W_{0}^{1,2}(\Omega) \hookrightarrow L^{p}(\Omega)$, then $\left(X,\|\cdot\|_{1},\|\cdot\|_{2}\right)$ is a binormed space such that $\|.\|_{2}$ is finer than $\|$. $\|_{1}$. Set $\mathrm{g}(\mathrm{u})=|u|^{\varepsilon+2}$ and consider the functional $G$ defined on $X$ by: $G(x)=\int_{\Omega} g(x(s)) d s$, then $G$ is $\|\cdot\|_{2}$-twice Fréchet differentiable at every $x \in X$. Moreover, $G^{1}(x) h=\int_{\Omega} g^{\prime}(x(s)) h(s) d s$, and $G^{2}(x)\left(h_{1}, h_{2}\right)=\int_{\Omega} g^{\prime \prime}(x(s)) h_{1}(s) h_{2}(s) d s . \quad G$ is $\left(\|\cdot\|_{1},\|\cdot\|_{2}\right)$-Fréchet strictly differentiable at every $x \in X$ and is not $\|$. $\|_{1}$-Fréchet strictly differentiable at any point $x$ of $X$, see [4]. Then our result given in Theorem 7 is better adapted for this example than the one given by Mordukhovich and Shao using the limiting subdifferential involving the norm $\|.\|_{1}$ in [6].

\section{References}

[1] F.H. Clarke, Optimization and Nonsmooth Analysis, Wiley (1983).

[2] J. Hlal, Subdifferentiation of marginal functions in binormed spaces, Intern. J. Math. Anal., 55 (2015), 2725-2732.

[3] J. Hlal, On strong limiting subdifferential calculus in separable binormed spaces, Rend. Circ. Mat. Palermo II, 66 (2017), 439-445.

[4] S. Lahrech, J. Hlal, A. Jaddar, A. Ouahab, A. Mbarki, On nonconvex subdifferential calculus in binormed spaces, Int. Math. Forum, 55 (2007), 2723-2731.

[5] S. Lahrech, J. Hlal, A. Jaddar, A. Ouahab, A. Mbarki, Characterizaion of the extrema of a pseudoconvex function in terms of limiting and strong limiting subdifferential, Int. Math. Forum, 55 (2007), 2711-2718.

[6] B.S. Mordukhovich, Y. Shao, On nonconvex subdifferential calculus in Banach spaces, J. Conv. Anal., 2 (1995), 211-227. 
